\title{
Nondimensional analysis of ballistic impact on thin woven laminate plates
}

\author{
Shirley K. García-Castillo, Sonia Sánchez-Sáez*, Enrique Barbero \\ Department of Continuum Mechanics and Structural Analysis, University Carlos III of Madrid, Avda. de la Universidad 30, 28911 Leganés, Madrid, Spain
}

\begin{abstract}
A B S T R A C T
In this work a nondimensional formulation of an analytical model was used to study the ballistic behaviour of composite plates made from woven laminates of E glass fibres. From this model the influence of two nondimensional ratios (the geometry and density ratios) at the ballistic limit, contact time, and energy absorption mechanisms were analysed at different impact velocities. The increase in both ratios raises the ballistic limit and varies the contact time between the projectile and plate. At impact velocities that do not cause perforation, the contact time decreases while at impact velocities over the ballistic limit, this time increases. Regardless of the ratio, the main energy absorption mechanism is the fibre failure at velocities below the ballistic limit, and the cone movement for impact velocities over the ballistic limit. At the ballistic limit the main mechanisms are fibre failure and fibre elastic deformation.
\end{abstract}

Ballistic impact

Analytical

Laminate plate

Absorbed energy

\section{Introduction}

Woven fabric laminates made with glass fibres are commonly used, due to their excellent energy absorption capabilities, in structures that should be subjected to ballistic impact, such as debris striking, fan blade impact or projectile hits. In these cases, analyses of the ballistic behaviour are needed to understand the condition for perforation by the projectile into the composite structure and the associated energy absorption mechanisms [1].

The perforation of a laminate structure is difficult to model due to the large number of parameters involved in this phenomenon [2-4]. In a woven laminate structure, the ballistic response depends on the mechanical properties and fabric structure of the laminate as well as on the physical properties of the projectile and plate $[1,5]$. Also, the kinetic energy of the projectile can be reduced by different mechanisms, such as elastic deformation of the lami nate, damage generation in the laminate (failure of fibres, delami nation, matrix cracking, etc.), inertia of the plate or back face deformation of the plate [6,7].

Several basic approaches are used to analyse the impact problem on composite structures: experimental, analytical, and numerical methods. Many works examine the impact behaviour of composite laminates by experimental tests [8-12]. Such tests are costly and time consuming and are valid only for the configuration

\footnotetext{
* Corresponding author. Tel.: +34 916248882; fax: +34 916248331. E-mail addresses: ssanchez@ing.uc3m.es, sgcastil@ing.uc3m.es (S. Sánchez-Sáez). URL: http://www.uc3m.es/mma
}

(plate and projectile) tested, since a change in a single parameter requires new tests. Numerical simulations have been successfully applied for modelling the perforation process of composite struc tures subjected to ballistic impact [7,13-15]. Nevertheless, such approaches require considerable computing time. Analytical models can be useful in providing a sufficiently accurate solution with a lower computational cost than that of the numerical methods. These methods solve the basic equation of Mechanics as well as simple constitutive relationships of the materials involved, taking into account the main physical aspect of the impact event [16]. They are very useful in optimisation processes because they serve to evaluate in a simple way the influence of the different parameters in the overall response of the structure [17].

There is continued interest in developing analytical models to predict the ballistic limit of laminate plates. This parameter is useful to define the condition of perforation of a structure, and can be defined as the maximum velocity at which a particular projectile is expected to consistently fail to penetrate the component [18]. Many of these models are based on energy conservation laws $[1-3,6,19,20]$ and others are based on momentum equations [4,16,21-23]. Several authors analyse the influence of a single parameter on the ballistic limit: the projectile diameter or projec tile mass [1], or the plate thickness [2,7,14,22,24,25]. Some authors show a non linear relationship between the ballistic limit and plate thickness, for E glass [2,24], kevlar [14], or carbon [25] laminates, using hemispherical, conical or flat faced projectiles. By contrast, Zhu et al. [22] affirm that there is a linear relationship between the ballistic limit and thickness for a Kevlar/polyester laminate under impact by flat faced projectiles. 


\begin{tabular}{|c|c|c|c|}
\hline \multicolumn{2}{|c|}{ Nomenclature } & \multirow{3}{*}{$\begin{array}{l}R_{C} \\
R_{l}\end{array}$} & \multirow{3}{*}{$\begin{array}{l}\text { distance covered by the transversal wave generated by } \\
\text { the impact on the plate or the cone radius } \\
\text { distance covered by the longitudinal wave generated } \\
\text { by the impact on the laminate }\end{array}$} \\
\hline$a$ & deceleration of projectile & & \\
\hline$B$ & yarn width & & \\
\hline$b$ & stress wave transmission factor & $r$ & distance from the impact point in the laminate plane \\
\hline$D$ & total distance travelled by the projectile or the depth of & $r_{p}$ & radius of projectile \\
\hline$d$ & the cone & $t_{c}$ & contact time between projectile and laminate during \\
\hline E & $\begin{array}{l}\text { alstance travelled by the projectile } \\
\text { Young modulus }\end{array}$ & $v$ & velocity of the projectile \\
\hline$E_{D L}$ & energy absorbed by delamination damage & $v_{l}$ & velocity of the longitudinal elastic waves \\
\hline$E_{E D}$ & energy absorbed by elastic deformation of fibres & $v_{0}$ & impact velocity of projectile \\
\hline$E_{F L}$ & energy absorbed by the failure of the laminate & $x$ & distance from impact point in the thickness direction \\
\hline$E_{K C}$ & kinetic energy of the cone & $x$ & distance from the impact point in the thickness \\
\hline$E_{M C}$ & energy absorbed by matrix cracking & & direction \\
\hline$E_{M T}$ & energy absorbed by matrix cracking per unit volume & $\Delta t$ & time interval \\
\hline$E_{T}$ & total energy absorbed by laminate & $\varepsilon_{f}$ & failure strain \\
\hline$E_{T F}$ & energy absorbed by fibre failure & $\Pi_{D R}$ & density ratio \\
\hline$e$ & laminate thickness & $\Pi_{G R}$ & geometry ratio \\
\hline$G_{I I_{C D}}$ & critical dynamic strain energy release rate in mode II & $\rho_{L}$ & laminate density \\
\hline$m_{c}$ & mass of the cone formed on the back side of the plate & $\rho_{p}$ & projectile density \\
\hline
\end{tabular}

Although some authors have analysed the influence of the ratio between projectile diameter and plate thickness [2,14], no systematic study is available on the influence of the parameters that control the impact process. One possible approach to this kind of study is to express the equations of a model in a nondimensional form.

The purpose of the present study is to analyse the parameters that affect the ballistic behaviour of woven laminate plates of $\mathrm{E}$ glass fibres, and to determine their influence on the ballistic limit, contact time, and the energy absorption mechanisms. A formula tion in nondimensional variables of the model proposed by the authors in a previous work [20] is used.

\section{Model desrcription}

The analytical model of García Castillo et al. [20] was devel oped for plain woven laminates of glass fibres in polymer resin, which allows the estimation of the residual velocity of the projectile, the ballistic limit, the energy absorbed by different mechanisms during the penetration of the laminate, and the contact time between the projectile and the laminate. This model is based on energy criteria and considers three energy absorption mechanisms: the elastic deformation of the fibres, the movement of a cone formed on the back side of the plate, and the generation of damage in the laminate. This latter mechanism included includes the failure of fibres and matrix as well as the damage by delamination.

In the formulation of the model, the following hypotheses were considered:

- The projectile is spherical, perfectly rigid and remains totally indeformable during impact.

- A moving cone is formed on the back side of the plate.

- The energy absorbed by shear plugging and friction are considered negligible.

- The energies absorbed by fibre elastic deformation and fibre failure are treated independently.

In this work that model was modified using a nondimensional formulation. The symbol $\wedge$ over any variable indicates non dimensionality. The following criteria were applied:
- Distances through plate thickness were divided by the plate thickness.

- Transversal distances from the impact point were divided by the projectile radius.

- The time was divided by a characteristic time defined as:

$t_{c} \frac{e}{\sqrt{\frac{E}{\rho_{L}}}} \frac{e}{v_{l}}$

- The mass of the cone was divided with the mass of projectile.

- Velocities were divided by the impact velocity.

- Energies were divided by the impact energy of the projectile.

When the equations of the model were expressed in a nondi mensional form using these criteria, two ratios appeared in a natural way:

Geometry ratio : $\Pi_{G R} \frac{e}{r_{p}}$

Density ratio : $\Pi_{D R} \frac{\rho_{l}}{\rho_{p}}$

The energy at an instant of time can be formulated as follows,

$\hat{E}_{T_{i}} \quad \hat{E}_{K C_{i}}+\hat{E}_{E D_{i}}+\hat{E}_{F L_{i}} \quad 1$

Where the energy absorbed by damage from the failure of the laminate is determined as

$\hat{E}_{F L_{i}} \quad \hat{E}_{T F_{i}}+\hat{E}_{D L_{i}}+\hat{E}_{M C_{i}}$

The different energy absorption mechanisms are described in a previous work [20].

To calculate the kinetic energy of the cone, it is assumed that the cone moves at the same velocity as the projectile. Therefore, this energy is: 
$\hat{E}_{K C_{i}} \quad \hat{m}_{c_{i}} \cdot\left(\hat{v}_{i}\right)^{2}$

The mass of the cone is defined by:

$\hat{m}_{c_{i}} \quad \frac{3}{4} \cdot\left(\hat{R}_{C_{i}}\right)^{2} \cdot \Pi_{G R} \cdot \Pi_{D R}$

The energy absorbed by tensile failure of fibres each instant of time is:

$\hat{E}_{T F_{i}} \quad \frac{3}{4} \cdot \sum_{1}^{j} \frac{\left(\hat{v}_{l}\right)^{2} \cdot \hat{d}_{j} \cdot\left(\Pi_{G R}\right)^{2} \cdot \Pi_{D R} \cdot \varepsilon_{f}^{2} \cdot \hat{B}}{\pi} \cdot \int_{0}^{2 \hat{R}_{l_{j}}} b^{\frac{2 \cdot \hat{x} \cdot \Pi_{G R}}{\hat{B}}} \cdot \mathrm{d} \hat{x}$

The computation of the energy absorbed by elastic deformation of fibres was made using the following equation:

$\left.\hat{E}_{E D i} \quad \frac{3}{2} \cdot \Pi_{G R} \cdot \Pi_{D R} \cdot\left(\hat{v}_{l}\right)^{2} \cdot \int_{1}^{\hat{R}_{c_{i}}}\left(\frac{\left(\hat{R}_{c_{i}}\right.}{\hat{r}}\right)\right)^{2} \cdot \hat{r} \cdot \mathrm{d} \hat{r}$

The contribution of the damage by delamination and damage from matrix cracking at the absorption of kinetic energy of projectile is associated with the area inside of the cone formed on the back of laminate. Two variables, nondimensional strain energy release rate and nondimensional cracking energy, were defined as:

$\hat{G}_{I I_{C D}} \frac{G_{I I_{C D}}}{r_{p} \cdot \rho_{p} \cdot v_{0}^{2}}$

$\hat{E}_{M T} \quad \frac{E_{M T}}{\rho_{p} \cdot v_{0}^{2}}$

The energy absorbed due to delamination and matrix cracking was calculated with the following equations:

$$
\begin{array}{ll}
\hat{E}_{D L_{i}} & \frac{3}{2} \cdot \hat{G}_{I I_{C D}} \cdot\left(\hat{R}_{C_{i}}\right)^{2} \\
\hat{E}_{M C_{i}} & \frac{3}{2} \cdot \hat{E}_{M T} \cdot \Pi_{G R} \cdot\left(\hat{R}_{C_{i}}\right)^{2}
\end{array}
$$

The velocity of the projectile at each instant of time can be estimated from Eqs. (4-6), as follows:

$\hat{v}_{i} \quad \sqrt{\frac{1}{1} \hat{E}_{F L_{(i 1)}}} \frac{3 / 4 \cdot \hat{R}_{c_{i}} \cdot \Pi_{G R} \cdot \Pi_{D R}}{1}$

This equation was solved numerically. It is assumed that the velocity of the projectile remains constant in each time integration interval .Therefore, the acceleration and the displacement of the projectile between two instances in time, $t_{i-1}$ and $t_{i}$, can be esti mated as:

$$
\begin{array}{ll}
\hat{a}_{i} & \frac{\hat{v}_{i-1} \hat{v}_{i}}{\Delta \hat{t}} \\
\hat{d}_{i} & \frac{1}{\hat{v}_{l}} \cdot \Delta \hat{t} \cdot\left(\frac{1}{2} \cdot \hat{v}_{i-1}+\hat{v}_{i}\right)
\end{array}
$$

The total displacement of the projectile at an instant of time can be determined as:

$\hat{D}_{i} \quad \sum \hat{d}_{i}$

\section{Model verification}

\subsection{Experimental validation}

The precision of the model in nondimensional variables was evaluated by the comparisons of the residual velocity and contact time for several impact velocities, and the ballistic limit with experimental data from previous works. The data used was from high velocity impact tests on E glass/polyester plain woven lami nates, with a geometry ratio of 0.85 and 1.60 , impacted by spherical steel projectiles $7.5 \mathrm{~mm}$ in diameter [12,20]. Table 1 shows the mechanical properties of this laminate, which registered at high strain rates $[1,26,27]$.

As can be seen in Fig. 1, the residual velocity estimated by the model agrees well with the values measured in the experimental test for both ratios analysed.

The contact time is defined, for velocities above ballistic limit, as the time necessary to perforate the laminate; and for impact velocities below the ballistic limit, as the time required for the projectile velocity to slow to zero. Fig. 2 shows the contact time estimated for the two geometry ratios analysed; also good agree ment with experimental data was found.

In Table 2, the ballistic limit estimated by the model is compared with experimental results, the differences between the two values being less than $6 \%$.

\subsection{Nondimensional verification}

For verification that the ratios selected in this study are truly nondimensional, several values of projectile radius, projectile density, laminate thickness, and laminate density were selected. Three geometry and density ratios were calculated from four different pairs of values. The ballistic limit was calculated and the results are shown in Tables 3 and 4 . Slight differences for each pair of values were found in the ballistic limit; for the geometry ratio the difference was below of $1.5 \%$ and for the density ratio is below of $5.8 \%$.

\section{Results}

An analysis was made concerning the influence of two nondi mensional variables - geometry ratio and density ratio- on the ballistic limit, on the contact time between projectile and laminate, and on the absorbed energy by each mechanism. These variables are present in several energy absorption mechanism equations, as can be seen in Eqs. (6-13).

Table 1

Mechanical properties of the laminate used in the model verification.

\begin{tabular}{ll}
\hline Properties & \\
\hline$E(\mathrm{GPa})$ & 15.2 \\
$\varepsilon_{f}$ & 0.072535 \\
$G_{I_{C D}}\left(\mathrm{~J} / \mathrm{m}^{2}\right)$ & 3000 \\
$b$ & 0.9 \\
$E_{M T}\left(\mathrm{~J} / \mathrm{m}^{3}\right)$ & $10^{6}$ \\
\hline
\end{tabular}


The variation range of these ratios was selected taking into account the following:

- The maximum thickness of laminate that can be used in the model was $7.5 \mathrm{~mm}$, because the analytical model was verified up to this thickness.

- The model applies only to plain woven laminates made from glass fibres, because to model the impact on laminate made with other fibres would require the consideration of other energy absorption mechanisms -for example, in carbon fibre laminates, shear plugging would need to be considered.

- The range of projectile density was selected from the density of a polymer to the density of lead.

From these considerations, the variation range of the geometry ratio was from 0.1 to 2 and the variation range of the density ratio was from 0.1 to 1.5 .
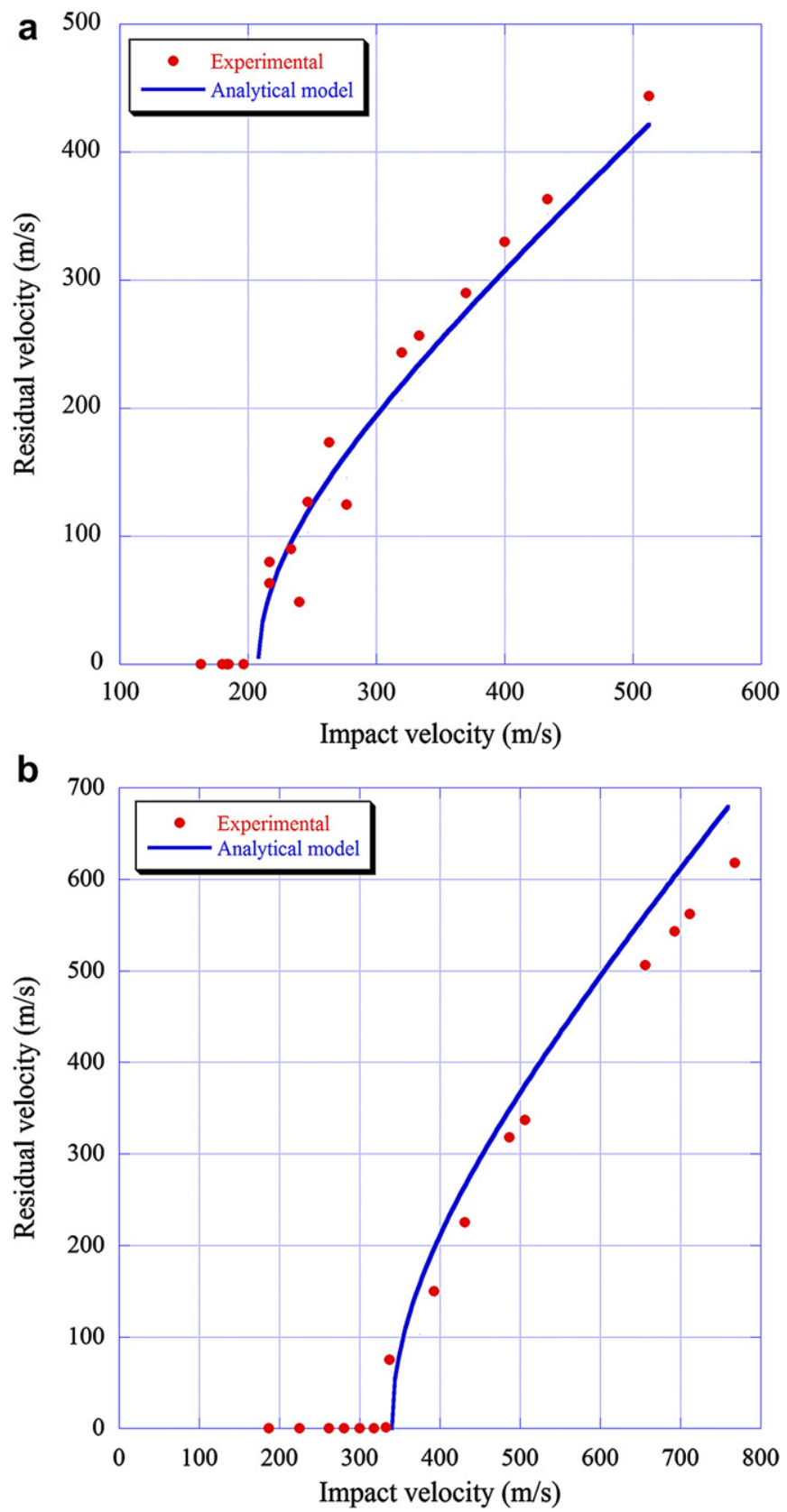

Fig. 1. Residual velocity vs. impact velocity for a geometry ratio of: a) 0.85 and b) 1.60 .
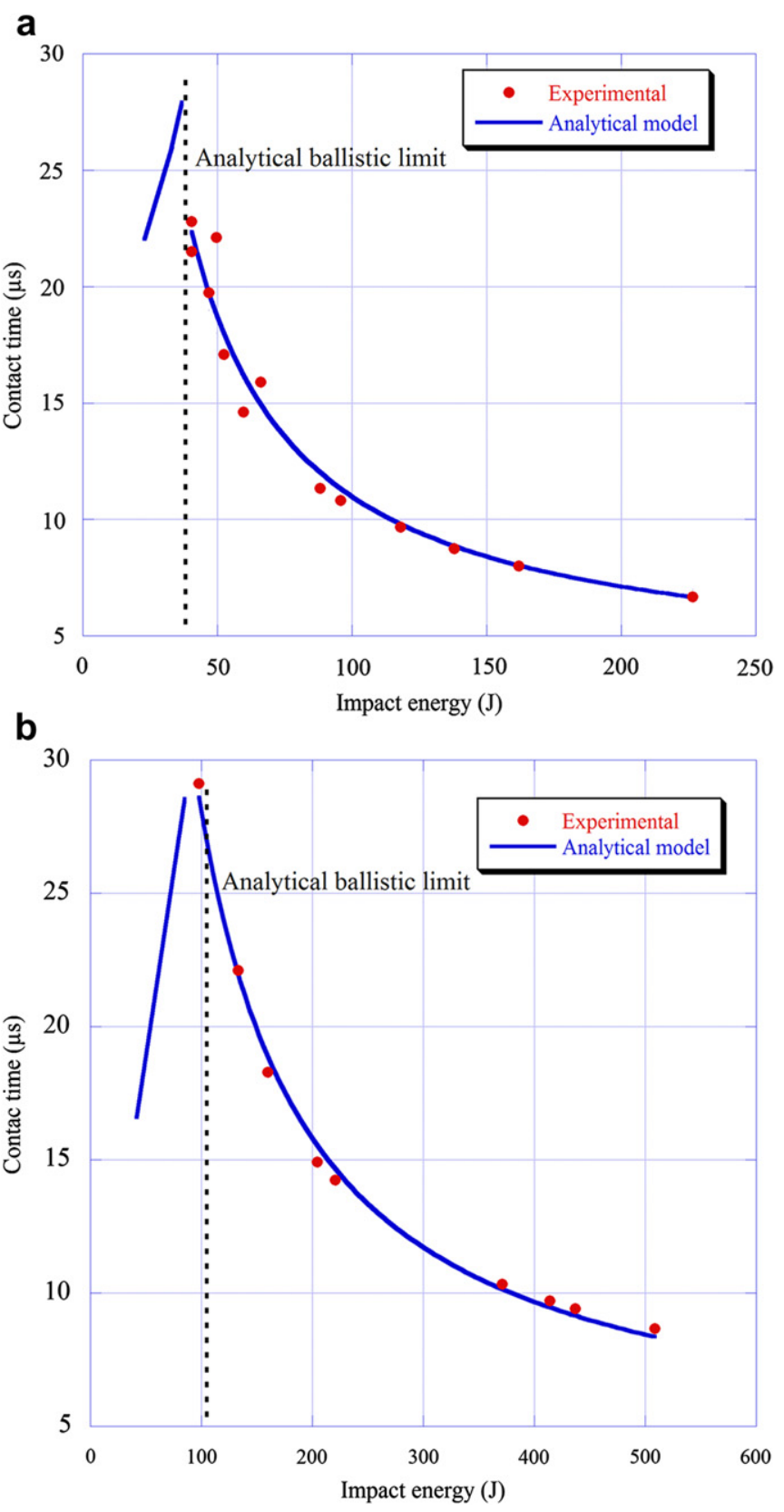

Fig. 2. Contact time vs. impact energy for a geometry ratio of: a) 0.85 and b) 1.60 .

To analyse the variation in the energy absorbed by each mech anism with the nondimensional ratios, several impact velocities were selected. First an impact velocity that did not result in the perforation for the entire range of the ratios considered, and second a impact velocity that resulted in perforation. In addition, impact velocities equal to the ballistic limit were chosen because the behaviour observed at these velocities differed from the others.

Table 2

Ballistic limit estimated by the model and calculated from the experimental tests for the two geometry ratios analysed.

\begin{tabular}{|c|c|c|c|c|}
\hline \multicolumn{2}{|c|}{ Laminate } & \multicolumn{2}{|c|}{ Ballistic limit (m/s) } & \multirow[t]{2}{*}{ Differences (\%) } \\
\hline$\overline{\Pi_{G R}}$ & $\overline{\Pi_{D R}}$ & Experimental & $\overline{\text { Analytical model }}$ & \\
\hline 0.85 & 0.25 & $212[20]$ & 208 & 1.9 \\
\hline 1.60 & 0.25 & $332[12]$ & 352 & 5.7 \\
\hline
\end{tabular}


Table 3

Ballistic limit for different geometry ratios, projectile radius and laminate thickness.

\begin{tabular}{lllll}
\hline $\begin{array}{l}\text { Geometry } \\
\text { ratio }\end{array}$ & $\begin{array}{l}\text { Projectile radius } \\
(\mathrm{mm})\end{array}$ & $\begin{array}{l}\text { Laminate thickness } \\
(\mathrm{mm})\end{array}$ & $\begin{array}{l}\text { Ballistic limit } \\
(\mathrm{m} / \mathrm{s})\end{array}$ & $\begin{array}{l}\text { Differences } \\
(\%)\end{array}$ \\
\hline 0.25 & 3.75 & 0.94 & 86 & 1.1 \\
& 8 & 2 & 85 & \\
& 15 & 3.75 & 84 & \\
& 30 & 7.5 & 84 & 1.4 \\
1 & 0.94 & 0.94 & 245 & \\
& 2 & 2 & 241 & \\
& 3.75 & 3.75 & 239 & \\
2 & 7.5 & 7.5 & 237 & \\
& 0.47 & 0.94 & 447 & \\
& 1 & 2 & 438 & \\
& 1.88 & 3.75 & 434 & \\
& 3.75 & 7.5 & 432 & \\
\hline
\end{tabular}

\subsection{Influence of the geometry ratio}

The variation of the ballistic limit with the geometry ratio is shown in Fig. 3. An increase in this ratio, and therefore in the thickness of the laminate or a decrease in projectile diameter, raised the ballistic limit. The data adjusted well to a straight line.

The relationship between the contact time and the geometry ratio was analysed for two impact velocities (Fig. 4). For a velocity that results in plate perforation $(800 \mathrm{~m} / \mathrm{s})$, the contact time increased with the geometry ratio as an inverse square root. However, for a velocity that does not lead to plate perforation $(30 \mathrm{~m} / \mathrm{s})$, the contact time decreases asymptotically to $6.25 \mu \mathrm{s}$, approximately. This latter relationship can be fit to a second degree polynomial.

The influence of the geometry ratio in the energy absorbed by each of the mechanisms studied in this model is shown in Fig. 5.

For impact velocities below the ballistic limit, the main energy absorption mechanism is fibre failure, while above the ballistic limit it is cone movement. This latter mechanism is negligible for velocities below the ballistic limit; however for those above, the least relevant mechanisms are fibre elastic deformation, delami nation, and matrix cracking.

In both cases the energy absorbed by fibre failure increases with the geometry ratio, since this energy is directly proportional to this ratio (Eq. (8)).

The energy absorbed by fibre elastic deformation diminishes to negligible values when the geometry ratio increases at impact velocities that do not perforate the plate. This energy absorption mechanism is controlled by the cone radius (Eq. (9)) that is directly proportional to contact time. As reflected in Fig. 4, this time diminishes with the geometry ratio.

At impact velocities over the ballistic limit the energy absorbed by cone movement increases with the geometry ratio. This

Table 4

Ballistic limit for different density ratios, laminate densities and projectile densities.

\begin{tabular}{lllll}
\hline $\begin{array}{l}\text { Density } \\
\text { ratio }\end{array}$ & $\begin{array}{l}\text { Laminate } \\
\text { density } \\
\left(\mathrm{kg} / \mathrm{m}^{3}\right)\end{array}$ & $\begin{array}{l}\text { Projectile } \\
\text { density } \\
\left(\mathrm{kg} / \mathrm{m}^{3}\right)\end{array}$ & $\begin{array}{l}\text { Ballistic } \\
\text { limit } \\
(\mathrm{m} / \mathrm{s})\end{array}$ & $\begin{array}{l}\text { Differences } \\
(\%)\end{array}$ \\
\hline 0.1 & 1600 & 16000 & 173 & 5.8 \\
& 1800 & 18000 & 159 & \\
0.7 & 2000 & 20000 & 155 & 5.5 \\
& 1600 & 2286 & 324 & \\
& 1800 & 2571 & 306 & 5.6 \\
& 2000 & 2857 & 290 & \\
& 1600 & 1067 & 418 & \\
& 1800 & 1200 & 394 & \\
& 2000 & 1333 & 374 & \\
\hline
\end{tabular}

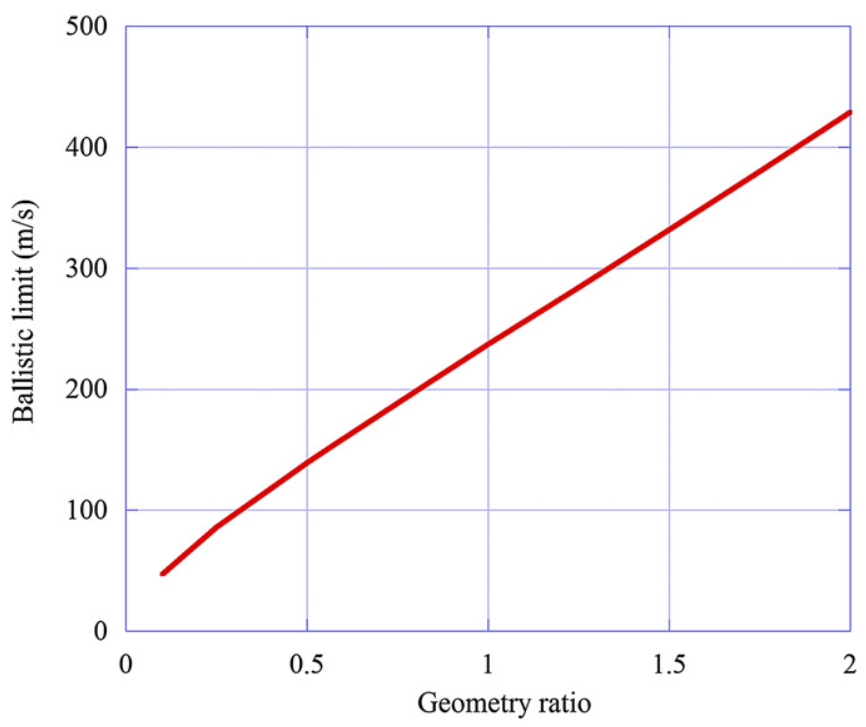

Fig. 3. Variation of the ballistic limit with the geometry ratio.

behaviour is caused by the increment of the mass of the cone (Eq. (6)), due to the rise in the cone radius and geometry ratio (Eq. (7)).

The cone radius increases because it is proportional to the contact time, which increases with the geometry ratio (Fig. 4).

At impact velocities that do not cause perforation, the energy absorbed by delamination diminishes with the geometry ratio, whereas that absorbed by matrix cracking increases. Both energies are proportional to the square of the cone radius (Eqs. (12) and (13)), which diminishes; whereas the second is also proportional to the geometry ratio. From the observed behaviour of the energy absorbed by matrix cracking, it can be deduced that the influence of the cone radius is less relevant than of the geometry ratio.

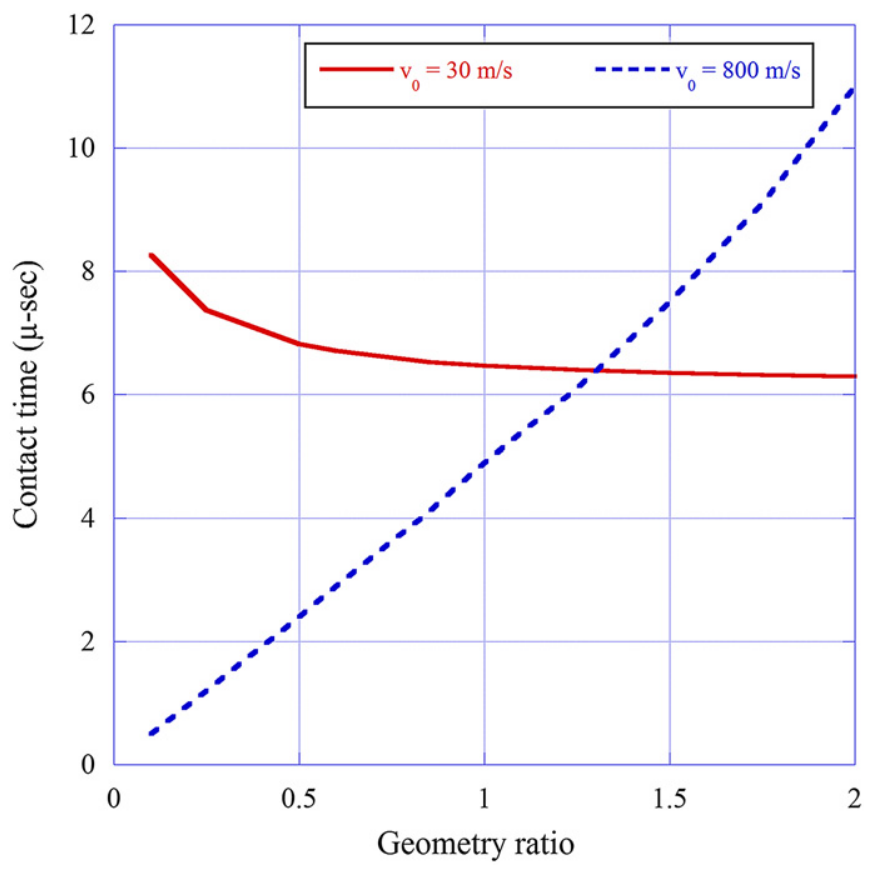

Fig. 4. Variation of the contact time between the projectile and the plate with the geometry ratio. 

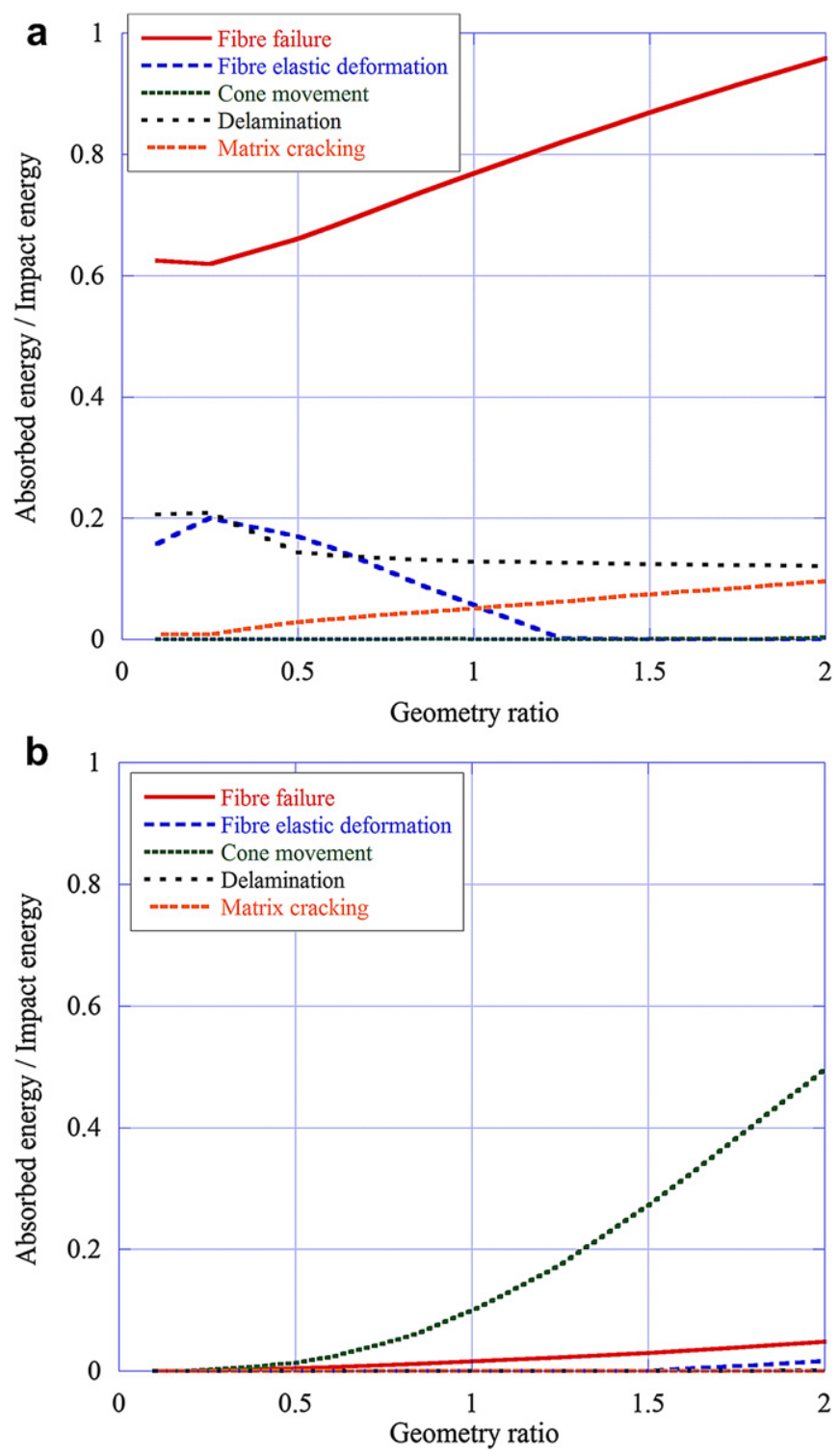

Fig. 5. Absorbed energy by different mechanism vs. geometry ratio at an impact velocity: a) below the ballistic limit, $30 \mathrm{~m} / \mathrm{s}$, and b) above the ballistic limit, $800 \mathrm{~m} / \mathrm{s}$.

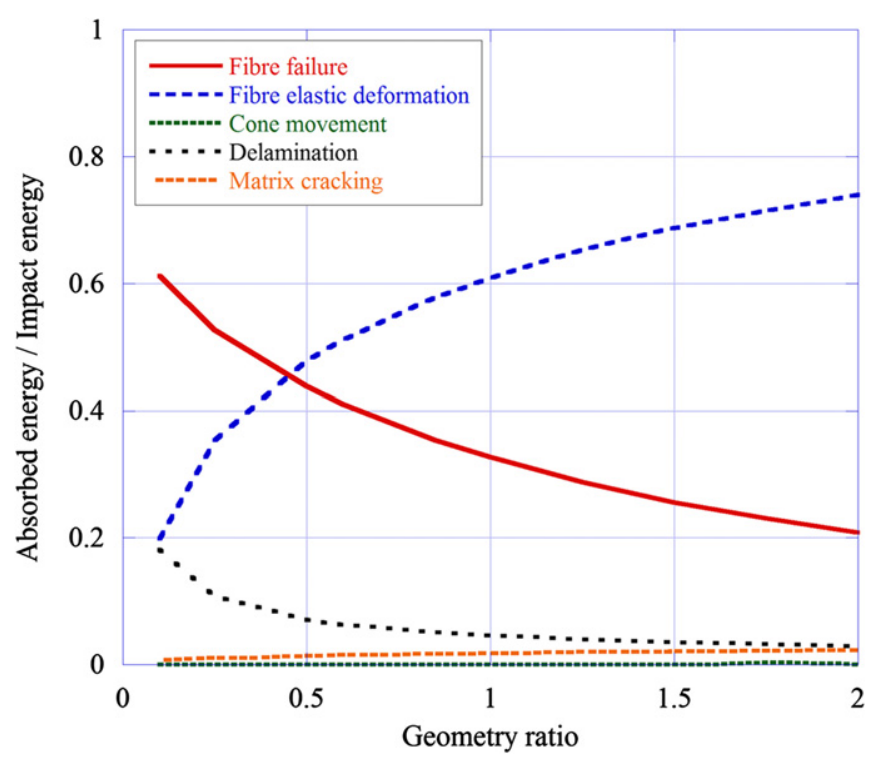

Fig. 6. Energy absorbed by different mechanisms vs. geometry ratio at the ballistic limit

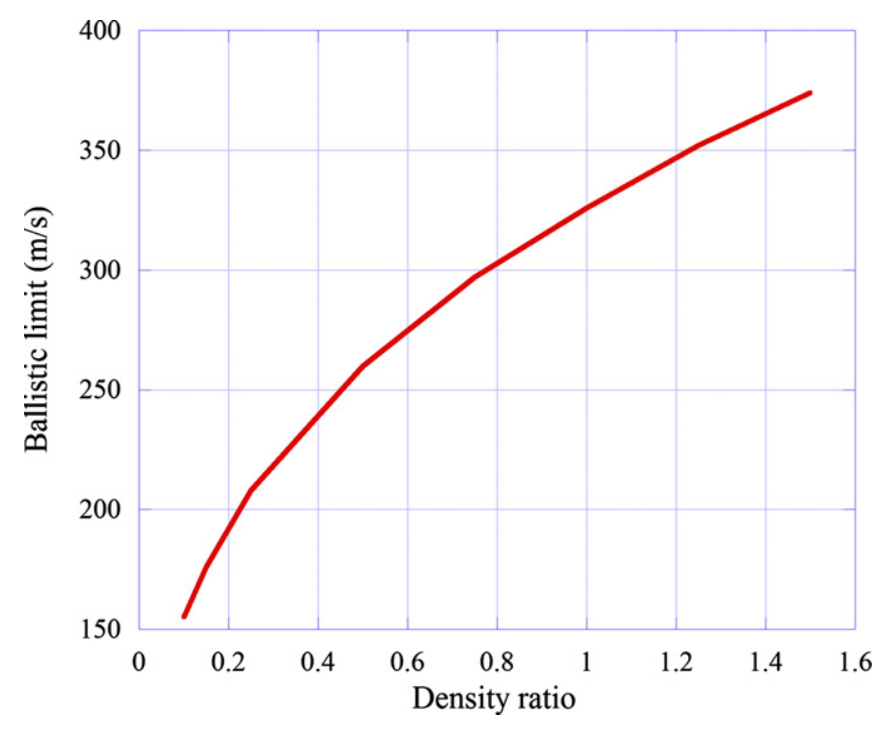

Fig. 7. Variation in the ballistic limit with the density ratio.

Also, the influence of geometry ratio in the energy absorption mechanisms for an impact velocity equal to the ballistic limit was analysed (Fig. 6). The ballistic limit is different for each value of that ratio (Fig. 3).

The main mechanisms of energy absorption at the ballistic limit are the fibre failure and the fibre elastic deformation, while the least relevant mechanisms are cone movement and matrix cracking.

The energy absorbed by fibre failure decreases with the geom etry ratio. This mechanism is controlled by $\hat{v}_{l}$, which decreases as the impact velocity increases.

On the contrary, the energy absorbed by fibre elastic deforma tion and matrix cracking increases because these mechanisms are

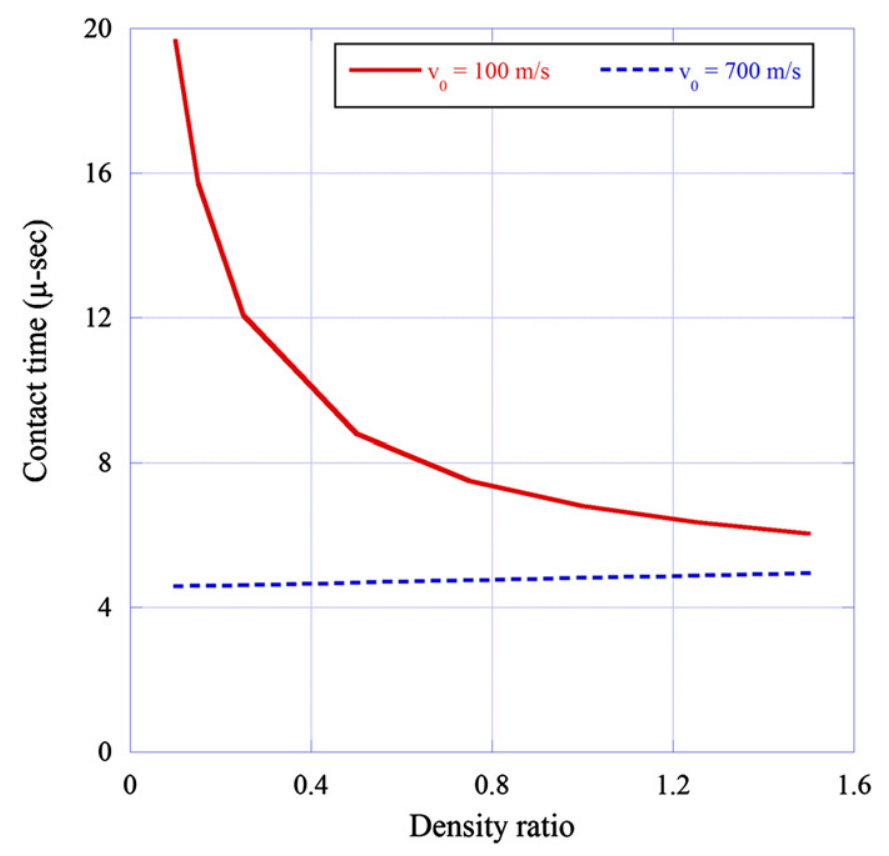

Fig. 8. Variation in the contact time between the projectile and the plate with density ratio. 
proportional to the geometry ratio and cone radius, which increase with contact time, as reflected in Eqs. (9 and 13).

The energy absorbed by delamination diminishes with the geometry ratio, because this mechanism depends on $\hat{G}_{I_{C D}}$ (Eq. (12)). The decrease is due to the fact that this variable is inversely proportional to the impact velocity squared (Eq. (12)).

\subsection{Influence of the density ratio}

The ballistic limit increases as a power law with the density ratio, Fig. 7.

The contact time and the absorbed energy for different mech anisms were calculated at an impact velocity $(100 \mathrm{~m} / \mathrm{s})$ that did not result in plate perforation, and a velocity $(700 \mathrm{~m} / \mathrm{s})$ that did cause plate perforation.

The variation in contact time with the density ratio is shown in Fig. 8 . The contact time increases lineally with this ratio for impact
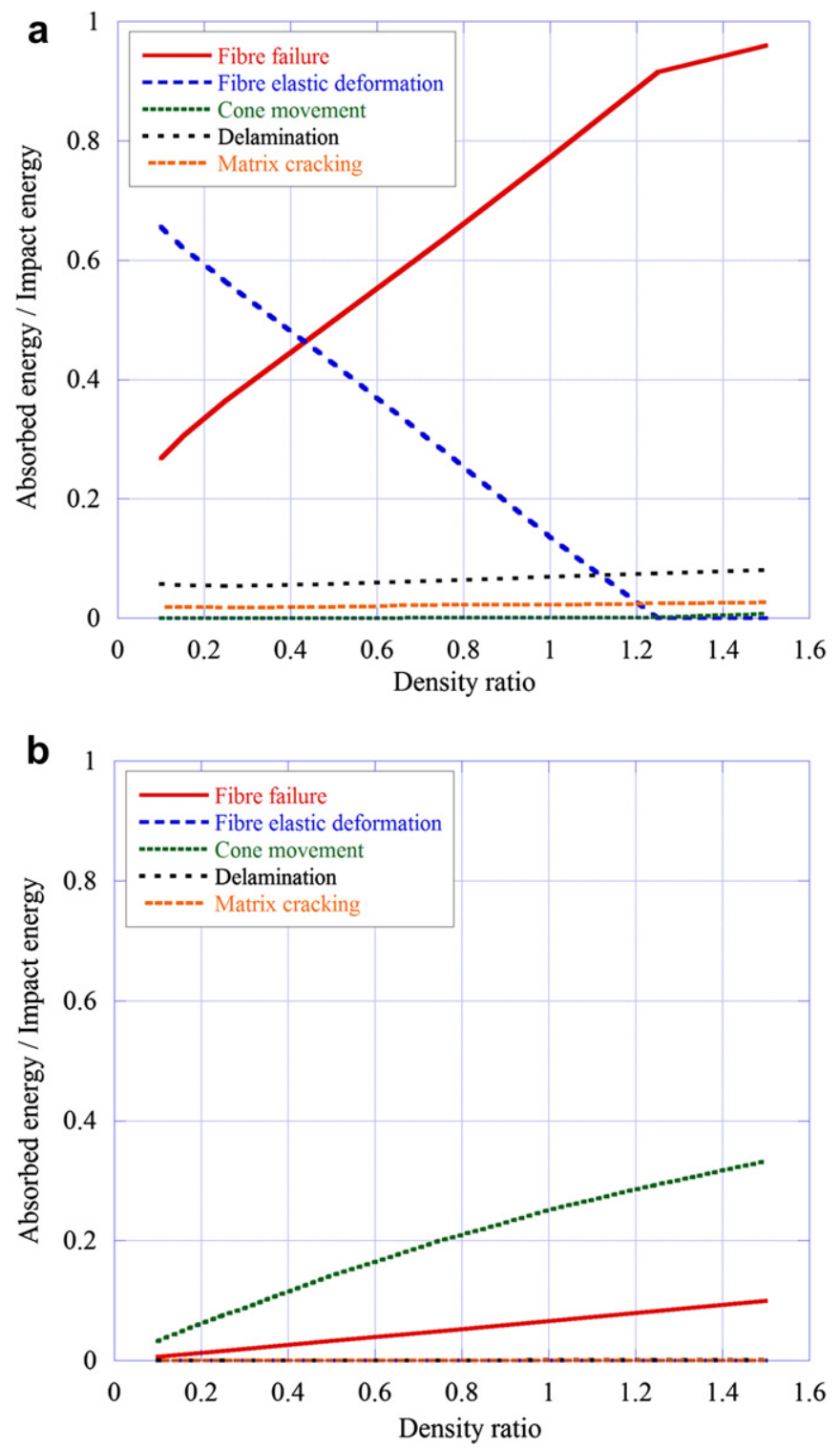

Fig. 9. Absorbed energy by different mechanisms vs. the density ratio at an impact velocity: a) below the ballistic limit, $100 \mathrm{~m} / \mathrm{s}$, and b) above the ballistic limit causes plate perforation, $700 \mathrm{~m} / \mathrm{s}$. velocities that cause plate perforation, diminishing for impact velocities below the ballistic limit. In the latter case the relationship can be fit to an inverse square root.

The main mechanisms of energy absorption at impact velocities below the ballistic limit are fibre failure and the fibre elastic deformation (Fig. 9a), while, for impact velocities above the ballistic limit, these mechanisms are the movement of the cone and the fibre failure (Fig. 9b).

The mechanism negligible for the velocities below the ballistic limit is cone movement, and for the those above are delamination, matrix cracking, and fibre elastic deformation.

In both cases, the energy absorbed by fibre failure increases with the density ratio, because the energy varies directly with this variable and $\hat{v}_{l}$ (Eq. (8)). Although $\hat{v}_{l}$ decreases with the density ratio, its influence on this mechanism is less relevant than this ratio.

The variation of energy absorbed by fibre elastic deformation and cone movement with the density ratio has a behaviour similar to that previously cited for the geometry ratio.

At impact velocities that do not perforate the plate (Fig. 9a), the energy absorbed by delamination and matrix cracking rise slightly with the density ratio, due to the increase of $\hat{G}_{I I_{C D}}$ and $\hat{E}_{M T}$ (Eqs. (12) and (13)).

The energy absorbed by the different mechanisms for impact velocities equal to ballistic limit vs. the density ratio is shown in Fig. 10.

The main mechanisms of energy absorption are fibre failure and fibre elastic deformation. The energy absorbed by fibre failure increases proportionally to the density ratio (Eq. (8)). On the contrary, the energy absorbed by the fibre elastic deformation decreases due to the influence of $\hat{v}_{l}$ (Eq. (9)), which diminishes with the density ratio and impact velocity (Eq. (1)). The impact velocity varies because the ballistic limit differs for each value of the ratio (Fig. 7).

The energy absorbed by cone movement increases slightly with the density ratio, due to the increased mass of the cone (Eq. (6)) This variable is proportional to density ratio and the cone radius squared (Eq. (7)).

Nevertheless, the energy absorbed by delamination and matrix cracking decreases slightly with the density ratio, caused by the reduction in $\hat{G}_{I I_{C D}}$ (Eq. (12)) and $\hat{E}_{M T}$ (Eq. (13)).

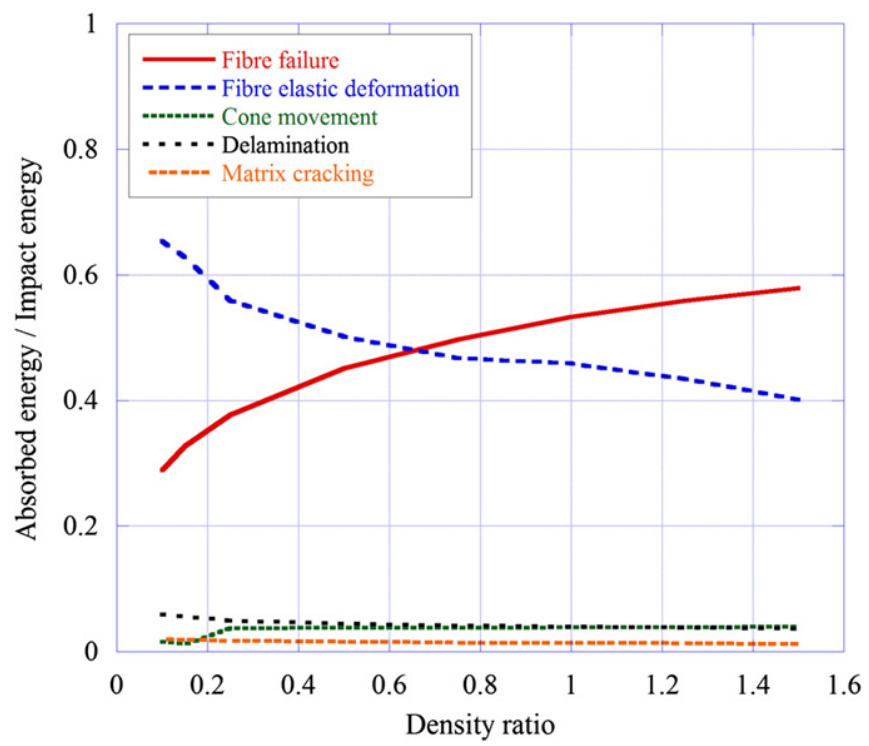

Fig. 10. Energy absorbed by different mechanisms vs. the geometry ratio at the ballistic limit. 


\section{Conclusions}

In this work a nondimensional formulation of an analytical model proposed in a previous work was developed. This formula tion can be used to estimate the evolution of projectile velocity and displacement during the perforation of thin plates made from a woven glass/polyester laminate. Also, the evolution of the energy absorbed by kinetic energy of the back cone, fibre failure, elastic deformation, matrix crack, and delamination were calculated.

The formulation identified the parameters which control the impact behaviour of a woven laminate plate. Two nondimensional ratios were defined: the geometry ratio and density ratio. It has been proved that these ratios are truly nondimensional in the range of values studied. The formulation was verified by experimental data for plates up to $7.5 \mathrm{~mm}$ in thickness. Good agreement was found for residual velocities, contact time and ballistic limit for two geometry ratios.

A rise in the ballistic limit was found when both ratios increased. A linear relationship was found between the ballistic limit and geometry ratio, and a power law between the ballistic limit and density ratio. The influence of both ratios in the contact time between projectile and plate proved similar. At impact below the ballistic limit the contact time decreased, and at impact velocities over the ballistic limit this time increased.

At impact velocities that did not cause plate perforation, the main energy absorption mechanism was fibre failure, regardless of the nondimensional ratio, and the cone movement, for impact velocities over the ballistic limit.

For impact velocities close to the ballistic limit the main mechanisms of energy absorption were the failure of primary fibres and the fibre elastic deformation.

\section{Acknowledgements}

The authors are indebted to the Spanish Comisión Interminis terial de Ciencia y Tecnología (Project TRA2007 66555) and Con sejería de Educación (Project GR/MAT/0498/2004) for the financial support of this work.

\section{References}

[1] Naik NK, Shrirao P, Reddy BCK. Ballistic impact behaviour of woven fabric composites: formulation. Int J Impact Eng 2006;32:1521 52.

[2] Wen HM. Penetration and perforation of thick FRP laminates. Compos Sci Technol 2001:61:1163 72 .

[3] Gu B. Analytical modelling for the ballistic perforation of planar plain-woven fabric target by projectile. Compos Part B-Eng 2003;34:361 71.
[4] Mamivand M, Liaghat GH. A model for ballistic impact on multi-layer fabric targets. Int J Impact Eng 2010;37:806 12.

[5] Cheeseman BA, Bogetti TA. Ballistic impact into fabric and compliant composite laminates. Compos Struct 2003;61:161 73.

[6] Morye SS, Hine PJ, Duckett RA, Carr DJ, Ward IM. Modelling of the energy absorption by polymer composites upon ballistic impact. Compos Sci Technol 2000;60:2631 42.

[7] Deka LJ, Bartus SD, Vaidya UK. Damage evolution and energy absorption of Eglass/polypropylene laminates subjected to ballistic impact. J.Mater Sci 2008; 43:4399 410.

[8] Fujii K, Aoki M, Kiuchi N, Yasuda E, Tanabe Y. Impact perforation behaviour of CFRPs using high-velocity steel sphere. Int J Impact Eng 2002;27:497 508 .

[9] Kim H, Welch DA, Kedward KT. Experimental investigation of high velocity ice impacts on woven carbon/epoxy composite panels. Compos Part A-Appl S 2003;34:25 41.

[10] Johnson AF, Holzapfel M. Influence of delamination on impact damage in composite structures. Compos Sci Technol 2006;66:807 15.

[11] García-Castillo SK, Sánchez-Sáez S, Barbero E, Navarro C. Response of preloaded laminate composite plates subject to high velocity impact. J Phys IV 2006;134:1257 63.

[12] Buitrago B, García-Castillo SK, Barbero E. Experimental analysis of perforation of glass/polyester structures subjected to high-velocity impact. Mater Lett 2010;64:1052 4 .

[13] Tan VBC, Ching TW. Computational simulation of fabric armour subjected to ballistic impacts. Int J Impact Eng 2006;32:1737 51.

[14] He T, Wen HM, Qin Y. Finite Element analysis to predict perforation and perforation of thick FRP laminates struck by projectiles. Int J Impact Eng 2008; $35: 2736$

[15] Buitrago BL, Navarro C, Sanchez-Sáez S, Barbero E, Navarro C. Modelling of composite sandwich structures with honeycomb core subjected to highvelocity impact. Compos Struct 2010;92:2090 6

[16] Navarro C. Simplified modelling of the ballistic behaviour of fabrics and fibrereinforced polymeric matrix composites. Key Eng Mater 1998;141-1:383 99.

[17] Santiuste C, Sanchez-Saez S, Barbero E. Application of the flexibility influence function method in the dynamic analysis of composite beams. Int J Solids Struct 2007; 44:4795 809 .

[18] MIL-STD-662F Standard. V50 Ballistic test for armor. Department Defense Test Method Stand.

[19] Lopez-Puente J, Zaera R, Navarro C. An analytical model for high velocity impacts on thin CFRPs woven laminated plates. Int J Solids Struct 2007;44: 283751.

[20] García-Castillo SK, Sánchez-Sáez S, López-Puente J, Barbero E, Navarro C. Impact behaviour of preloaded glass/polyester woven plates. Compos Sci Technol 2009;69:711 7.

[21] Taylor WJ, Vinson JR. Modeling ballistic into flexible materials. AIAA J 1990; 28:2098 103.

[22] Zhu G, Goldsmith W, Dharan CKH. Penetration of laminated Kevlar by projectiles-II. Analytical model. Int J Solids Struct 1992;29:421 36.

[23] Vinson JR, Walter JM. Ballistic impact of thin-walled composite structures. AIAA J 1997;35:875 8.

[24] Gellert EP, Cimpoeru SJ, Woodward RL. A study of the effect of target thicknesss on the ballistic perforation of the glass-fibre-reinforced plastic composites. Int J Impact Eng 2000;24:445 56.

[25] He T, Wen HM, Qin Y. Penetration and perforation of FRP laminates struck transversely by conical-nosed projectiles. Compos Struct 2007:81:243 52 .

[26] Harding J, Ruiz C. The mechanical behaviour of composite materials under impact loading. Key Eng Mater 1998;141-143:403 26.

[27] Ochola RO, Marcus K, Nurick GN, Franz T. Mechanical behaviour of glass and carbon fiber reinforced composite at varying strain rates. Compos Struct 2004; 63:455 67. 